Western University

Scholarship@Western

Paediatrics Publications

Paediatrics Department

$11-2017$

\title{
Canadian Guidelines for Controlled Pediatric Donation After Circulatory Determination of Death-Summary Report
}

Matthew J Weiss

Laura Hornby

Bram Rochwerg

Michael van Manen

Sonny Dhanani

See next page for additional authors

Follow this and additional works at: https://ir.lib.uwo.ca/paedpub

Part of the Pediatrics Commons

Citation of this paper:

Weiss, Matthew J; Hornby, Laura; Rochwerg, Bram; van Manen, Michael; Dhanani, Sonny; Sivarajan, V Ben; Appleby, Amber; Bennett, Mary; Buchman, Daniel; Farrell, Catherine; Goldberg, Aviva; Greenberg, Rebecca; Singh, Ram; Nakagawa, Thomas A; Witteman, William; Barter, Jill; Beck, Allon; Coughlin, Kevin; Conradi, Alf; Cupido, Cynthia; Dawson, Rosanne; Dipchand, Anne; Freed, Darren; Hornby, Karen; Langlois, Valerie; Mack, Cheryl; Mahoney, Meagan; Manhas, Deepak; Tomlinson, Christopher; Zavalkoff, Samara; and Shemie, Sam D, "Canadian Guidelines for Controlled Pediatric Donation After Circulatory Determination of Death-Summary Report" (2017). Paediatrics Publications. 112.

https://ir.lib.uwo.ca/paedpub/112 


\section{Authors}

Matthew J Weiss, Laura Hornby, Bram Rochwerg, Michael van Manen, Sonny Dhanani, V Ben Sivarajan, Amber Appleby, Mary Bennett, Daniel Buchman, Catherine Farrell, Aviva Goldberg, Rebecca Greenberg, Ram Singh, Thomas A Nakagawa, William Witteman, Jill Barter, Allon Beck, Kevin Coughlin, Alf Conradi, Cynthia Cupido, Rosanne Dawson, Anne Dipchand, Darren Freed, Karen Hornby, Valerie Langlois, Cheryl Mack, Meagan Mahoney, Deepak Manhas, Christopher Tomlinson, Samara Zavalkoff, and Sam D Shemie 


\title{
Canadian Guidelines for Controlled Pediatric Donation After Circulatory Determination of Death-Summary Report ${ }^{*}$
}

\author{
Matthew J. Weiss, MD, FRCPC ${ }^{1,2,3}$; Laura Hornby, $\mathrm{MSc}^{3}$; Bram Rochwerg, MD, MSc, FRCPC \\ Michael van Manen, MD, PhD, FRCPC5; Sonny Dhanani, MD, FRCPC ${ }^{6}$; \\ V. Ben Sivarajan, MD, MSc, FRCPC ${ }^{7}$; Amber Appleby, RN, MM³; Mary Bennett, MD, FRCPC; \\ Daniel Buchman, MSW, PhD ; Catherine Farrell, MD, FRCPC ${ }^{10}$; Aviva Goldberg, MD, MA, FRCPC ${ }^{11}$; \\ Rebecca Greenberg, RN, PhD ${ }^{12}$; Ram Singh, MD, FRCPC ${ }^{13}$; Thomas A. Nakagawa, MD, FAAP, FCCM ${ }^{14}$; \\ William Witteman, MIS ${ }^{15}$; Jill Barter, MD, FRCPC ${ }^{16}$; Allon Beck, MD, FRCPC ${ }^{17}$; \\ Kevin Coughlin, MD, MSHc, FRCPC ${ }^{18}$; Alf Conradi, MD, FRCPC ${ }^{19}$; \\ Cynthia Cupido, MD, MSc, FRCPC ${ }^{20}$; Rosanne Dawson, LLB ${ }^{21}$; Anne Dipchand, MD, FRCPC ${ }^{22}$; \\ Darren Freed, MD, PhD, FRCPC ${ }^{23}$; Karen Hornby, $\mathrm{MSc}^{3}$; Valerie Langlois, MD, FRCPC ${ }^{24}$; \\ Cheryl Mack, MD, MA, FRCPC ${ }^{25}$; Meagan Mahoney, MD, FRCPC ${ }^{26}$; Deepak Manhas, MD, FRCPC ${ }^{27}$; \\ Christopher Tomlinson, MD, FRCPC ${ }^{28}$; Samara Zavalkoff, MD, FRCPC ${ }^{29}$; \\ Sam D. Shemie, MD, FRCPC $3,30,31$
}

\begin{abstract}
*See also p. 1068.
${ }^{1}$ Division of Pediatric Intensive Care, CHU de Quebec, Centre MèreEnfant Soleil, Québec, QC, Canada.

${ }^{2}$ Department of Pediatrics, Université Laval Faculté de Médecine, Québec, QC, Canada.

${ }^{3}$ Canadian Blood Services Decreased Donation, Ottawa, ON, Canada.

${ }^{4}$ Department of Medicine \& Department of Clinical Epidemiology \& Biostatistics, McMaster University, Hamilton, ON, Canada.

${ }^{5}$ Division of Neonatal Intensive Care, University of Alberta \& Stollery Children's Hospitals, Edmonton, AB, Canada.

${ }^{6}$ Division of Critical Care, Children's Hospital of Eastern Ontario, Ottawa, ON, Canada.

${ }^{7}$ Division of Paediatric Cardiac Intensive Care Unit, University of Alberta \& Stollery Children's Hospitals, Edmonton, AB, Canada.

${ }^{8}$ Division of Critical Care, BC Children's Hospital, Vancouver, BC, Canada. ${ }^{9}$ Bioethics Program, University Health Network, Toronto, ON, Canada.

${ }^{10} \mathrm{Critical}$ Care Medicine, Département de pédiatrie, CHU Sainte-Justine, Montréal, QC, Canada.

${ }^{11}$ Department of Pediatrics and Child Health, Faculty of Medicine, University of Manitoba, Winnipeg, MB, Canada.

Copyright (c) 2017 The Author(s). Published by Wolters Kluwer Health, Inc. on behalf of the Society of Critical Care Medicine and the World Federation of Pediatric Intensive and Critical Care Societies. This is an open-access article distributed under the terms of the Creative Commons Attribution-Non Commercial-No Derivatives License 4.0 (CCBY-NC-ND), where it is permissible to download and share the work provided it is properly cited. The work cannot be changed in any way or used commercially without permission from the journal.

DOI: $10.1097 /$ PCC. 0000000000001320
\end{abstract}

${ }^{12}$ Department of Bioethics, Hospital for Sick Children, Toronto, ON, Canada.

${ }^{13}$ Division of Paediatric Critical Care Medicine, Western University Children's Hospital, London, ON, Canada.

${ }^{14}$ Division of Critical Care, Johns Hopkins All Children's Hospital, St. Petersburg, FL.

${ }^{15}$ Information Specialist, Québec, QC, Canada

${ }^{16}$ Division of Pediatric Intensive Care, Janeway Childrens Health and Rehabilitation Centre, St. Johns, NL, Canada.

${ }^{17}$ Division of Pediatric Intensive Care, Victoria General Hospital, Victoria, BC, Canada.

${ }^{18}$ Division of Neonatal Intensive Care, Children's Hospital of the London Health Sciences Centre, London, ON, Canada.

${ }^{19}$ Division of Pediatric Intensive Care, University of Alberta \& Stollery Children's Hospitals, Edmonton, AB, Canada.

${ }^{20}$ Division of Pediatric Critical Care, Hamilton Health Sciences, Hamilton, ON, Canada.

${ }^{21}$ Canadian Blood Services Legal Department, Ottawa, ON, Canada.

${ }^{22}$ Division of Pediatric Cardiology, Hospital for Sick Children, Toronto, ON, Canada.

${ }^{23}$ Division of Cardiac Surgery, University of Alberta Hospital, Edmonton, $\mathrm{AB}$, Canada.

${ }^{24}$ Division of Nephrology, Hospital for Sick Children, Toronto, ON, Canada. ${ }^{25}$ Division of Pediatric Anesthesia, Stollery Children's Hospital, Edmonton, AB, Canada.

${ }^{26}$ Division of Pediatric Intensive Care, Alberta Children's Hospital, Calgary, $\mathrm{AB}$, Canada.

${ }^{27}$ Division of Neonatal Intensive Care, BC Women's Hospital + Health Centre, Vancouver, BC, Canada.

${ }^{28}$ Division of Neonatology, Hospital for Sick Children, Toronto, ON, Canada. 
${ }^{29}$ Division of Critical Care, Montreal Children's Hospital, McGill University Health Centre and Research Institute, Montréal, QC, Canada.

${ }^{30}$ Division of Critical Care, Montreal Children's Hospital, McGill University Health Centre and Research Institute, Montréal, QC, Canada.

${ }^{31}$ Department of Pediatrics, McGill University, Montréal, QC, Canada.

This work was endorsed by the Canadian Paediatric Society, the Canadian Critical Care Society, the Canadian Association of Critical Care Nurses, and the Canadian Society of Transplantation.

This work was performed by Dr. Weiss in collaboration with the above authors at the $\mathrm{CHU}$ de Quebec, Centre Mère-Enfant Soleil, and the Université Laval, Québec, QC, Canada.

Financially supported by Canadian Blood Services. Funding from other sources reported by authors was for work outside of this particular project.

Dr. Weiss received funding from the Canadian Blood Services; he disclosed that he was recently nominated as medical director of Transplant Québec, a governmental nonprofit organ donation organization; and he disclosed government work. Dr. Hornby received funding from the Canadian Blood Services (paid research consultant and was paid for a portion of the work on this project). Dr. Sivarajan received funding from the Canadian Blood Services (hotel/travel for consensus meeting for guideline development) and from the Ontario Trillium Gift of Life Network (stipend paid for his role as hospital donation physician as part of provincial program). Dr. Appleby received funding from the Canadian Blood Services (employee where part of her paid time was spent supporting this work; sponsored travel and meeting costs associated with the face to face meetings). Dr. Farrell received funding from the Canadian Blood Services (payments made to defray travel expenses for attendance at two plenary meetings to plan the work and prepare the major portion of the article), Centre de soins de santé et de services sociaux des Laurentides (honorarium for lecture at a conference on head trauma organized by the health authority in May 2015), CHU Sainte-Justine Honorarium (lecture given during a Continuing Medical Education conference [Pédiatrie Plus] held outside the hospital, in November 2016), and the Canadian Paediatric Society (member of the Executive and Board of Directors of the Society, and a portion of her travel expenses are paid for attendance at Board meetings); and she disclosed that she is a designated organ donation physician specialist for her institution, and she works closely with Transplant-Québec. Dr. Goldberg received funding from the Canadian Blood Services (funding for travel to meetings for preparation of the article). Dr. Nakagawa received funding from UpToDate, and he disclosed that he is the Assistant Medical Director for Carolina Donor Services in Durham, NC. Dr. Barter received funding from Canadian Blood Services provided support for transportation to a meeting in October 2015 to commence the process of writing these guidelines. Dr. Dawson received funding from Canadian Blood Services (employer), and she disclosed work for hire. Dr. Dipchand's institution received funding from the National Institutes of Health/National Institute of Allergy and Infectious Diseases. Dr. Hornby received funding the Canadian Blood Services. Dr. Langlois' institution received funding from Astella Pharma Canada for a grant not related to the submitted work. Dr. Mack received funding for travel from the Canadian Blood Services. Dr. Mahoney received funding from the Canadian Blood Services. Dr. Shemie disclosed that he is a medical advisor for deceased organ donation at Canadian Blood Services, and he disclosed government work. The remaining authors have disclosed that they do not have any potential conflicts of interest.

Address requests for reprints to: Matthew J. Weiss, Département de pédiatrie Centre Mère-Enfant Soleil du CHU de Québec 2705 boul Laurier Local R1735 Québec, QC G1V 4G2; and Canadian Blood Services, Deceased Donation, 1800 Alta Vista Drive Ottawa, Ontario, K1G 4J5. E-mail: matthew-john.weiss@mail.chudequebec.ca

Objectives: Create trustworthy, rigorous, national clinical practice guidelines for the practice of pediatric donation after circulatory determination of death in Canada.

Methods: We followed a process of clinical practice guideline development based on World Health Organization and Canadian Medical Association methods. This included application of Grading of Recommendations Assessment, Development, and Evaluation methodology. Questions requiring recommendations were generated based on 1) 2006 Canadian donation after circulatory determination of death guidelines (not pediatric specific), 2) a multidisciplinary symposium of national and international pediatric donation after circulatory determination of death leaders, and 3) a scoping review of the pediatric donation after circulatory determination of death literature. Input from these sources drove drafting of actionable questions and Good Practice Statements, as defined by the Grading of Recommendations Assessment, Development, and Evaluation group. We performed additional literature reviews for all actionable questions. Evidence was assessed for quality using Grading of Recommendations Assessment, Development, and Evaluation and then formulated into evidence profiles that informed recommendations through the evidence-to-decision framework. Recommendations were revised through consensus among members of seven topic-specific working groups and finalized during meetings of working group leads and the planning committee. External review was provided by pediatric, critical care, and critical care nursing professional societies and patient partners.

Results: We generated 63 Good Practice Statements and seven Grading of Recommendations Assessment, Development, and Evaluation recommendations covering 1) ethics, consent, and withdrawal of life-sustaining therapy, 2) eligibility, 3) withdrawal of life-sustaining therapy practices, 4) ante and postmortem interventions, 5) death determination, 6) neonatal pediatric donation after circulatory determination of death, 7) cardiac and innovative pediatric donation after circulatory determination of death, and 8) implementation. For brevity, 48 Good Practice Statement and truncated justification are included in this summary report. The remaining recommendations, detailed methodology, full Grading of Recommendations Assessment, Development, and Evaluation tables, and expanded justifications are available in the full text report.

Conclusions: This process showed that rigorous, transparent clinical practice guideline development is possible in the domain of pediatric deceased donation. Application of these recommendations will increase access to pediatric donation after circulatory determination of death across Canada and may serve as a model for future clinical practice guideline development in deceased donation. (Pediatr Crit Care Med 2017; 18:1035-1046)

Key Words: clinical practice guidelines; deceased organ donation; donation after circulatory determination of death; end-of-life care; ethics

S ince the publication of the 2006 consensus Canadian recommendation (1), donation after circulatory determination of death (DCD) has become an increasingly frequent path to donation for adults (2). Implementation of pediatric DCD (pDCD) has lagged behind. According to 2014 data from Canadian Blood Services, DCD represented 21\% of total national deceased donation, but pDCD made up only $8 \%$ of pediatric deceased donation. The purpose of this document is to provide rigorously developed, evidence-based guidelines that centers can use to develop pDCD in Canada.

One of the methods proposed by donation experts to improve $\mathrm{pDCD}$ practice is standardization and evidence-based recommendations (3). Current $\mathrm{pDCD}$ practice varies by jurisdiction and center $(3,4)$, likely as a result of the fact that no 
national or international guidelines specifically address pDCD. As detailed below, we have employed a rigorous guideline development methodology, including an extensive literature review (5) and multidisciplinary consultation to create recommendations for all aspects of $\mathrm{pDCD}$.

\section{METHODS}

The guideline was developed by a multidisciplinary guideline development committee that included seven topic-specific working groups (WGs). Two patient-family partners, professional society partners, and an international expert provided external review. Funding was provided by Canadian Blood Services. No guideline development member disclosed any financial conflicts of interest with for-profit entities, though several were or are paid donor physicians associated with governmental notfor-profit organ donation organizations (ODOs), and others have active research and academic activities in organ donation.

The guideline development committee adhered to a rigorous development process based on the Grading of Recommendations Assessment, Development, and Evaluation (GRADE) methods and consistent with recommendations from several national and international bodies (6-9). The scope of the guideline included only controlled pDCD (e.g., after planned withdrawal of life-sustaining therapies [WLSTs]). Specifically, we defined uncontrolled pDCD, or donation after cardiac arrest outside of a WLST setting, to be outside the scope of these guidelines. The guideline development committee and WGs judged the quality of evidence and created evidence-todecision tables before making either "strong" or "conditional" recommendations according to the GRADE approach (10). In cases where the guideline development committee felt that there was insufficient evidence or the balance of benefits and harms was likely neutral, no recommendation was made.

In addition to GRADEd recommendations, the guideline development committee formulated Good Practice Statements (GPSs) in cases where there was a large body of indirect evidence strongly supporting the net benefit of the recommendation or there was no reasonable comparator (11). Full consensus by all guideline development committee and WG members was achieved for all recommendations.

This report is a summary that does not include all the recommendations or the justifications. For all recommendations, justifications, complete GRADE tables for actionable recommendations, and a comprehensive description of the guideline development process, please refer to the full report available at http://www.organsandtissues.ca/s/english-expert/leading-practices-public-awareness-and-education. For a global review of the $\mathrm{pDCD}$ literature, please refer to the associated scoping review (5).

\section{RECOMMENDATIONS}

\section{Ethics and WLST}

\section{Good Practice Statements.}

1) pDCD is a medically and ethically viable pathway to provide access to deceased organ donation.
2) The option of deceased donation, including pDCD, should be routinely incorporated into end-of-life (EOL) care.

3) Healthcare systems should establish processes to ensure pDCD access.

4) Throughout the WLST and donation process, healthcare professionals must respect the dignity of the dying process.

5) The discussions and process of deceased donation should respect the beliefs and values of the surrogate decision makers and other loved ones involved.

6) In recognition of diversity of perspectives on $\mathrm{PDCD}$, healthcare professionals should be allowed to conscientiously object to participation in pDCD.

a) In the case of healthcare professional objection, institutions should work to honor the surrogate decision makers' wishes to donate.

Justification. The option to offer DCD as part of EOL care is universally supported by professional societies and ODOs that have examined the issue $(1,12-15)$, including two specific endorsements from the American Academy of Pediatrics $(16,17)$. Despite this broad consensus, some authors have expressed concerns around ethical aspects of pDCD $(18,19)$. Some individuals within the healthcare team may have differing views on the meaning and permissibility of deceased organ and tissue donation (OTD) based on societal, cultural, religious, and other personal beliefs (20). These concerns justify the above recommendation to allow conscientious objection by healthcare professionals to not participate in $\mathrm{pDCD}$, consistent with other policy and position statements $(12,17,21)$. However, considering the important role donation can play in the lives of donor families, these objections should not prohibit substitute decision makers and families from participating in $\mathrm{pDCD}$ if they so desire, which is why we emphasize that institutions should work to accommodate these requests using the principles of effective referral.

\section{Decision-Making Process for WLST Good Practice Statements.}

7) The decision to pursue WLST must not be influenced by donation potential and should proceed according to accepted medical practices.

8) The ODO, organ recovery, and transplant team must not be involved in the decision to pursue WLST or have direct contact with surrogate decision makers before WLST decisions are finalized.

a) Treating teams may contact ODOs to assess eligibility prior to the decision to pursue WLST, as long as there is no direct contact between the ODO and surrogate decision makers.

9) The decision to pursue WLST should be made before any discussion of OTD that is initiated by healthcare professionals.

b) If surrogate decision makers initiate organ donation discussions prior to the decision to pursue WLST, information may be provided, but consent discussions should be deferred until WLST decisions have been finalized. 
10) Safeguards should be in place to ensure that mitigation of conflict of interest for the case where a patient who is a potential donor and a patient who is a potential recipient are being cared for in the same care unit.

Justification. In order to avoid real or perceived conflicts of interest, decisions pertaining to OTD must be kept as separate as possible from decisions regarding WLST. As is universally supported in the literature, the above recommendations support that WLST decision-making follow established, best practices regardless of pDCD potential $(12-14,16,17,22-26)$.

One area we believe merits particular attention is when a patient who is a potential donor and a patient who is a potential recipient are simultaneously cared for in the same unit. This possibility is more likely in pediatric than adult practice given the smaller number of recovery and transplant hospitals. We acknowledge this as a potential conflict and encourage systems to ensure ethical safeguards if the substitute decision maker is motivated to pursue donation in this setting. Measures to mitigate this potential conflict will depend on local context but could include ethics consultation or a second opinion from an uninvolved clinician.

\section{Eligibility}

\section{Good Practice Statements.}

11) Individual transplant programs, in collaboration with pediatric and neonatal healthcare professionals and ODOs, should determine criteria for donor eligibility, limits of warm, and cold ischemic time. Special consideration should be given for neonatal patients who are potential donors.

12) Coroners must be notified prior to donation proceedings according to provincial laws. If coroner evaluation and approval to pursue $\mathrm{pDCD}$ is required, this should be done prior to consent discussions with the surrogate decision makers.

Justification. We chose to limit our recommendations related to pDCD eligibility. Further national recommendations will require input from multidisciplinary groups, including transplant surgeons and physicians caring for recipients of $\mathrm{pDCD}$ organs, in order to form organ-specific recommendations. Current recommendations from groups such as the Canadian Society of Transplantation should inform these discussions, including those on high-risk donors (27). These criteria will be subject to change based on center experience, further research, and recommendations from organ-specific transplantation groups.

\section{Consent for pDCD}

\section{Good Practice Statements.}

13) Consent discussions for pDCD can include members of the care team, representatives of the ODO, or healthcare professionals from both groups.

a) As stated in GPS 9 above, all discussions of organ donation initiated by healthcare providers must be deferred until after WLST decisions are finalized.
14) The person or team discussing consent should have extensive knowledge of the local process and should clearly identify their institutional affiliations.

15) Consent conversations with surrogate decision makers should include the opportunity to discuss beliefs and values around all aspects of pDCD, including death and death determination.

16) At minimum, the following information should be provided to surrogate decision makers regarding the $\mathrm{pDCD}$ process:

a) Logistics of the process, including that WLST may be delayed due to pDCD logistics, and where WLST will occur,

b) The procedures and methods of determining death, including that these practices conform to accepted medical and legal standards,

c) Which organs are potentially eligible for recovery,

d) That consenting for $\mathrm{pDCD}$ does not guarantee organ recovery or transplantation,

e) If organ recovery is not possible, tissue donation may remain an option,

f) How EOL care would proceed if they decline organ donation or if recovery does not occur after attempted donation,

g) That the treating team has no influence over allocation, which may include allocation to adult or pediatric recipients,

h) That surrogate decision makers will be supported if they consent to or decline pDCD, and

i) That consent can be withdrawn at any time, including after the determination of death.

17) Tests and interventions prior to death (antemortem interventions) to facilitate donation in $\mathrm{pDCD}$ require specific and informed consent from the surrogate decision makers for each intervention.

a) Antemortem interventions should only be undertaken with disclosure and consideration of risks and benefits to the patient who is a potential donor.

b) Antemortem interventions should not be intended to hasten death.

c) Antemortem interventions should pose no more risk to the patient than routine intensive care practices.

18) Antemortem interventions should be recognized as providing nonmedical benefit to the patient who is a potential donor by allowing realization of interest and intent to donate despite the fact that these interventions provide no medical benefit to the patient who is a potential donor. This justifies surrogate decision maker's authority to consent to interventions that pose no increased risk beyond routine intensive care practices despite no medical benefit to the patient who is a potential donor.

Justification. How best to engage in consent discussions was carefully considered for these recommendations. There is significant practice variability concerning which healthcare 
professionals should be present during consent discussions. Regardless of whether an ODO representative is present during the consent request, we recommend that person have detailed knowledge of the local processes and procedures. Further discussion of training requirements for people requesting consent can be found under Actionable Recommendation 1. Furthermore, the stage in the EOL pathway at which ODOs are to be notified varies across jurisdictions, often related to requirements of mandatory reporting laws. The laws and local practices of reporting of a patient who is a potential donor should be carefully considered when establishing a pDCD protocol.

The understanding of consent for $\mathrm{pDCD}$ also requires an understanding of the distinction between consent for interventions before (ante) and after (post) mortem. It is outside the scope of these guidelines to extensively review the legal framework governing pDCD consent, but deceased donation in Canada is governed by provincial tissue gift legislation. Similar to the concept of "authorization" used more commonly in the United States, permission to proceed with donation under gift acts is different from, and legally less demanding than informed consent for treatment of a living patient (28). Consideration of benefit or harm posed to the patient, which forms the basis of informed consent to treatment, cannot be applied in the context of postmortem organ recovery any more than it can applied to the processes of cremation or embalming (28).

pDCD, however, includes both authorization for postmortem organ recovery and consent for antemortem interventions that do require full informed consent. A question that is often raised in $\mathrm{pDCD}$ considerations is if substitute decision makers or families can give valid consent for a procedure that might cause harm or discomfort to the donor while providing medical benefit only to the organ recipient. Several authors (22, 29, 30), including the 2013 American shared position statement from the American Thoracic Society, the Society for Heart and Lung Transplantation, the Society of Critical Care Medicine, the Association of Organ Procurement Organizations, and the United Network of Organ Sharing (12), answer in the affirmative. Their rationale is that if the process presents potential risk of harm that is similar to routine intensive care practices, and the procedure is in line with parental values, an assumption of altruism is legitimate $(22,29)$. The benefit to the patient who is a potential donor is therefore allowing donation to proceed in order to fulfill family or surrogate desire to donate, and it is this benefit that justifies assumption of risk without direct medical benefit. This is consistent with the ethical reasoning supporting children's participation in medical research where there is no hope for direct benefit to them. Furthermore, not allowing patients' substitute decision makers or families to accept this level of risk in order to act altruistically in this circumstance would limit their autonomy (21). These arguments, however, are not universally accepted, and others claim that altruism on the part of an incompetent child cannot be assumed based on parental values (18). We conclude that divergent opinions regarding the ethical acceptability of such antemortem interventions would be a justifiable reason for healthcare professionals to excuse themselves from $\mathrm{pDCD}$ proceedings through conscientious objection.
It is impossible to specifically recommend how to weigh these risks and benefits in all the nuanced situations that will arise for individual patients (e.g., heparin administration to a patient with a remote history of an intracranial hemorrhage). It is the responsibility of the treating team, with appropriate ethical oversight, to ensure that protection of the interests of the patient who is a potential donor remains the primary concern throughout these situations.

Any test of organ eligibility or those specific for allocation (e.g., human leukocyte antigen matching) should be considered an antemortem test and should not be performed until consent for the $\mathrm{PDCD}$ process and required investigations has been obtained.

For further information regarding general best practices in organ donation consent, please consult the recent report from Canadian Blood Services (31).

Actionable Recommendation. "Should trained professionals versus professionals without specific training be used for approaching families for consent in the setting of pediatric donation after circulatory death?"

19) The panel did not make a recommendation regarding minimal required training of professionals approaching families for consent in the setting of pediatric donation after circulatory death.

Justification. Although several observational reports (32-43) suggest that trained requesting is effective at increasing consent rates, the only randomized controlled trial (44) showed no effect of involving trained ODO staff at the time of consent. None of these studies were exclusive to pediatrics or even DCD. Only one (33) of 13 references examined family satisfaction after the consent process as an outcome.

Considering the lack of conclusive evidence supporting benefit, and the substantial system investment that would be required to have trained requesters present at every consent conversation, we chose to not recommend for or against this intervention. For further information on effective requesting techniques in deceased donation, please refer to the recently published report from Canadian Blood Services (31).

\section{Procedures for WLST in the Context of PDCD Good Practice Statements.}

20) WLST practice should be based on established ICU or hospital practices, policies, and guidelines.

21) The critical care team must be responsible for patient management between the decision to WLST and the determination of death.

22) The ODO, organ recovery, and transplant team must not be involved in any aspect of management of the dying process.

23) WLST may occur in the critical care unit, near the operating room, or in the operating room, as determined by surrogate decision makers' preferences, institutional logistics, resources, and facilities. 
24) Psychosocial, spiritual, and bereavement support should be provided to surrogate decision makers regardless of WLST location.

25) Wherever WLST occurs, surrogate decision makers and other loved ones should be given the option to be physically present with the patient who is a potential donor until the determination of death is complete.

26) The organ recovery team should not be physically present in the room until the determination of death has been completed and the surrogate decision makers are escorted from the bedside.

27) If a patient who is a potential donor is hospitalized where pDCD is not available, and the surrogate decision makers are motivated to donate, consideration should be given for patient transfer to a hospital that performs $\mathrm{pDCD}$.

Justification. The fiduciary responsibilities of ICU clinicians are first and foremost to act in the best interest of his or her patient $(12,13,16)$, and we therefore strongly support that in the event of a conflict in management goals between organ donation and optimal EOL care, care for the dying child should always take precedence. As universally supported in the published literature, WLST practices should be provided with minimal deviations from standard practice, including full support available for families (12-14, 16, 17, 21, 22, 25, 26, 45 ). Our group did not specifically deliberate on the issue of whether the practice of surgical preparation of the patient who is a potential donor (sterilization of the surgical field, draping, etc.) represented acceptable contact between the recovery team and the donor prior to death determination. In line with other recommendations, if this practice is required by the recovery team, it should be treated as an antemortem intervention requiring specific informed consent, and the recovery team should in no way otherwise influence EOL care. Also if practiced, this contact should not alter the recommendation that the recovery team not be physically present in the room at the time of death determination, and they should leave the operating room after surgical preparation is completed and before the parents enter.

\section{Time From WLST to Determination of Death Good Practice Statements.}

28) A maximum time limit from the start of WLST to death, beyond which organs will not be recovered, should be established in collaboration with ODOs and local transplant teams.

Justification. The duration of acceptable warm ischemic time (WIT) should be locally informed and based on organspecific concerns $(13,46,47)$. Current practice in most pDCD centers recommends WIT of 30-90 minutes, depending on the organ to be recovered. Adult practices may vary from 1 to 4 hours depending on multiple factors, including limitations of access to operating rooms. Our guideline committee did not consist of transplant surgeons or posttransplantation physicians who could provide meaningful expertise into the effects of various WIT thresholds on specific organs, and we therefore specifically chose not to make recommendations regarding the length of acceptable WIT.

Actionable Recommendation. "Should formal predictive tools versus no formal tool (clinical judgment) be used for predicting time of death within 30 or 60 minutes of WLST?"

29) The panel did not make a recommendation regarding use of tools to predict the time from WLST to death.

Justification. Though a prediction tool developed by Shore et al (48) has shown reasonable predictive value, it remains to be tested against clinical judgment or prospectively validated. Prediction tools cause no direct harm to a patient, may provide important information to the clinical team and surrogate decision makers, and are low cost. The risk, however, is if clinicians choose whether or not to pursue donation proceedings based solely on such a tool without understanding its strengths and limitations. Although future iterations may result in improved sensitivity and specificity, we currently do not recommend for or against the use of death prediction tools.

\section{Minimum Standards Required for Death Determination in PDCD}

Definition of Death Used for these Recommendations. There is currently no Canadian federal, provincial, or territorial statute mandating how clinicians determine when a patient is dead. As there is also no widely accepted medical standard from Canadian professional societies, we have chosen, for the purposes of this guideline, to use the following definition taken from recently proposed guidelines at the World Health Organization:

The definition of death by circulatory determination: The permanent loss of capacity for consciousness and all brainstem functions, as a consequence of permanent cessation of circulation. Permanence is defined as loss of function that will not resume spontaneously and will not be restored through intervention (49).

This definition is consistent both with current accepted Canadian medical practice and the definitions used in the current clinical practice recommendations governing donation circulatory death in adults (1).

Good Practice Statements. The following includes a summary of current Canadian laws and practices governing deceased donation. These laws and recommendations should be understood to represent the minimum standards necessary to determine death. They do not preclude additional standards, as long as those standards are accepted prior to implementation by all stakeholders.

30) The dead donor rule must be respected within the context of $\mathrm{pDCD}$.

31) Death must be determined by two physicians in accordance with accepted medical practice.

a) The two physicians must confirm their determinations concurrently at the end of a hands-off period of observation during circulatory arrest. 
32) No physician who has active involvement in transplant procedures or allocation of donated organs shall take any part in donor death determination.

33) The minimum level of physician qualification required to determine death in PDCD is as follows:

a) They possess the requisite skills and training. A particular level of specialty certification is not required, but skills and training should include ability to interpret monitoring used.

b) At least one of these physicians must be an attending physician staff in the ICU of the patient and possess full and current licensure for independent medical practice in the relevant Canadian jurisdiction.

c) The second physician could be on an educational register (e.g., residents, fellows), as long as they have the requisite skills and training.

34) The following criteria must be met before organ recovery:

a) Circulatory arrest, defined as the absence of anterograde arterial circulation. See actionable recommendations 37 and 38 for the specifics of how to determine that absence.

b) A hands-off period of continuous observation of circulatory arrest during which no interventions are undertaken to facilitate donation. See recommendation below for duration of hands-off period.

c) At the end of this period, death is legally determined, and organ recovery may commence.

35) Recovery and transplantation of the heart in pDCD is consistent with the dead donor rule, as death is based on the permanent cessation of circulation.

36) The same criteria should apply to all potential pDCD donors including those undergoing withdrawal of mechanical circulatory support such as extracorporeal mechanical oxygenation (ECMO).

Justification. The definition of death used for these guidelines represents both current accepted Canadian practice and is consistent with evolving international consensus (49). The details of how cessation of circulation is determined and for how long are detailed below in actionable recommendations 37 and 38. pDCD, practiced according to these practices and definitions, respects the dead donor rule, defined as "vital organs should only be taken from dead patients and, correlatively, living patients must not be killed by organ retrieval" (50).

We have chosen to recommend that although the first physician determining death in pDCD must have a full, unrestricted license to practice, the second may be a trainee on an educational register. This recommendation considers that the death determination in pDCD requires skills or training that would be readily available to a resident or fellow undergoing training in a PICU or neonatal ICU (NICU). If the second physician is on an educational register, he or she should be reminded that they are not obligated to participate and that a decision to participate or no will not affect their evaluation. Also, the second physician need not be from a certain specialty, as long as he or she possesses the capacity to determine death in this setting, specifically the ability to interpret an arterial catheter waveform tracing.

In GPS 32, active involvement in transplant procedures or allocation is defined as any involvement in postmortem surgical recovery procedures, discussions of which patient on the transplant wait list will receive the donated organs, or participation in any part of the transplantation procedure (including anesthesia of the recipient). Consultant physicians who might be involved in evaluating the patient for donor eligibility and might also care for a potential recipient (e.g., nephrologists) would be excluded from participating in the determination of death. As addressed in GPS 10, it is possible that intensive care physicians will be involved directly or indirectly with the care of both donor patients and those who receive transplanted organs from the same donor due to the limited number of PICUs in Canada. Ethical safeguards should be developed in these cases.

Regarding GPS 36, in the past, when DCD was more commonly referred to as "donation after cardiac death," authors argued that determining death by irreversible loss of cardiac function precluded DCD cardiac transplantation $(51,52)$. However, our guidelines specifically define death as permanent loss of circulation in the donor. Whether the heart remains unresuscitated in the donor or is removed and resuscitated in another patient does not alter donor outcome: body and brain circulation remains permanently ceased in the dead donor (53). Thus, cardiac pDCD practiced according to these guidelines would respect the dead donor rule.

Actionable Recommendation. "Should arterial line versus palpable pulses and auscultation be used for confirmation of lack of anterograde circulation?"

37) We recommend that a well-functioning arterial catheter be used to confirm arrest of anterograde arterial circulation for the determination of death. (strong recommendation, low certainty in evidence)

Justification. Although not specific to a pDCD setting, data from studies designed to test clinicians ability to determine between low and nonpulsatile states suggest that even experienced PICU physicians commit errors (54-56). The panel strongly felt that palpation of pulse was an inadequate method to confirm lack of circulation. Arterial catheter monitoring is commonly used, easily interpreted, and objective. The recommendation to rely on arterial catheter monitoring assumes a functioning and verified arterial catheter. No other confirmation of loss of anterograde circulation (e.g., electrical asystole) is necessary when a well-functioning arterial catheter is in place. Although auscultation or palpation should not be used to confirm lack of circulation, they could be applied to verify that an observed flat waveform corresponds with the clinical state. We make no recommendation as to the required site of the arterial catheter.

Please see the full report for consideration of situations when an arterial catheter is not possible for technical reasons or due to surrogate decision maker refusal, including the use of other modalities such as echocardiography. 
Actionable Recommendation. "Should 10 minutes handsoff time versus 5 minutes hands-off time be used for death determination in pDCD donors?"

38) We suggest 5 minutes of hands-off observation of arrest of circulation prior to determination of death. (conditional recommendation, very low certainty in evidence)

Justification. Currently, all Canadian adult and pediatric centers performing DCD use 5-minute hands-off period. During this 5-minute period, also sometimes referred to as a "no touch" period, no healthcare professional should have any physical contact with the patient, and the physicians determining death should be constantly observing the method used to confirm absent circulation. The period of time commences when no visible pulsatility is observable on a well-functioning arterial catheter waveform or after the last evidence of anterograde circulation (e.g., last opening of the aortic valve by echocardiography). If evidence of circulation is detected during the 5-minute hands-off period, the observation period should recommence until 5 full minutes of absent circulation are observed. This period is longer than any reported case of autoresuscitation after WLST $(57,58)$, and organs transplanted after this ischemic time have acceptable outcomes (59-61). However, based on the low quality of the reviewed autoresuscitation evidence and the fact that no reports compared organ outcomes using 5-versus 10-minute hands-off times, we chose to make a conditional recommendation.

\section{Ante and Postmortem Interventions}

\section{Good Practice Statements.}

Antemortem.

39) Any intervention or test that may pose discomfort to the patient who is a potential donor should be managed with analgesia and/or sedation as per standard ICU practices.

40) Consideration should be given to the timing of administration of any antemortem pharmacologic intervention in order to minimize any potential risks.

\section{Postmortem.}

41) Interventions that do or may reinstitute oxygenated brain blood flow after death must not be performed, including cyclic ventilation after reintubation for lung donation.

42) Only the organ recovery team may carry out postmortem surgical interventions.

Justification. The above recommendations emphasize that any antemortem intervention, including transfer of a patient who is a potential donor, carries the same requirements for informed consent, minimization of risk, and respect for the comfort of the patient as in routine care of ICU patients. Refer to the "Consent for Antemortem Interventions" section above for additional discussion.

Regarding postmortem interventions, our primary concern was the need to avoid interventions that might reestablish oxygenated brain blood flow. Absence of oxygenated brain blood flow is the key component of the determination of death, so procedures that potentially reestablish that flow could violate the dead donor rule. Understanding that risk, we recommend that tracheal reintubation is permissible as long as cyclic ventilation is not provided. Through cardiopulmonary interactions, cyclic ventilation has the theoretical risk of restoring oxygenation and brain circulation, and its avoidance has been recommended by other groups $(12,14)$.

Actionable Recommendation. "Should Heparin versus no anticoagulation be used for pDCD as an ante mortem intervention?"

43) The panel did not make a recommendation regarding the universal administration of heparin in the setting of pDCD.

Justification. Given the lack of available evidence for benefit in pediatric or adult patients $(62,63)$ and concerns regarding any antemortem interventions that could cause harm to a patient who is a potential donor, we make no recommendation regarding routine antemortem heparin administration pDCD. If given, practices such as dose and timing of administration should be determined jointly by intensive care teams, ODOs, and transplant programs to ensure that harm to a patient who is a potential donor is minimized.

Actionable Recommendation. "Should regional oxygenated perfusion techniques versus no such techniques be used for improving organ outcome in controlled pDCD?"

44) We recommend that regional perfusion not be used in the setting of pDCD (strong recommendation, very low certainty in evidence).

Justification. Although not practiced in Canada, other jurisdictions have employed perfusion techniques (e.g., modified ECMO) that provide oxygenated blood flow to abdominal organs after death but prior to organ recovery, while excluding blood brain flow. Reports of this practice were considered indirect to our question, since they were almost exclusive to adult donors (64-74), and often involved antemortem interventions such as cannulation that would be in conflict with current Canadian pDCD practice.

Given the low quality of the evidence reporting benefit, risk of the significant consequence of reestablishing brain blood flow through inadequate aortic occlusion, and the cost/ resources involved, we feel that regional perfusion techniques should not be used for $\mathrm{pDCD}(64,73)$.

Although we do not recommend its use in standard practice, regional perfusion techniques could be considered as part of a research protocol with research ethics board approval. Oversight should include techniques to ensure the absence of brain blood flow during regional perfusion.

Actionable Recommendation. "Should bronchoscopy versus no bronchoscopy be used for ante mortem evaluation of lung function in potential pDCD donors?"

45) The panel did not make a recommendation for or against the routine use of antemortem bronchoscopy in the setting of $\mathrm{pDCD}$. 
Justification. Although bronchoscopy is frequently practiced in neurologic determination of death and DCD organ donation, its association with graft or recipient outcome in $\mathrm{pDCD}$ is not well described (75-77). We acknowledge that antemortem bronchoscopy prior to controlled pDCD is likely a low risk procedure, but there are no published reports evaluating adverse events rates in this setting. This balance of considerations led us to not recommend for or against antemortem bronchoscopy.

The possibility of postmortem bronchoscopy either in situ or ex vivo is unreported in the current literature but would likely be of similar benefit as a pretransplant bronchoscopic evaluation while eliminating risk conferred to the patient who is a potential donor.

\section{Cardiac pDCD}

\section{Good Practice Statements.}

46) Considering the lack of published experience in cardiac pDCD:

a) Cardiac transplant programs should establish criteria for acceptance of heart donation, ex vivo cardiac protocols, and heart allocation in $\mathrm{pDCD}$,

b) Consideration should be given to initiate cardiac pDCD program as either research protocols with research ethics board oversight or through programs that oversee innovative therapies.

Justification. Although there is minimal published experience with cardiac pDCD (78), recent innovative reports of adult cardiac DCD using ex vivo heart preservation suggests that this option may evolve as a viable clinical pathway in the near future (79). We considered this lack of evidence when recommending that future Canadian cardiac pDCD should be undertaken under the supervision of a clinical trial or innovative therapy program.

\section{Neonatal DCD}

\section{Good Practice Statements.}

47) Unless otherwise stated, the above GPSs and actionable recommendations that apply to infants and children should also apply to neonates, provided expertise in neonatal EOL care can be provided.

48) Diagnoses such as anencephaly or other similar severe, lifelimiting neurologic disorders, for whom NDD is impossible, do not preclude consideration as potential candidates for $\mathrm{pDCD}$.

49) Centers not providing pDCD should establish a clear process for transfer to hospitals with pDCD programs including consideration of transfer of the mother of the patient who is a potential donor, ongoing provision of EOL care, limitation of economic burden on surrogate decision makers, and repatriation of the body.

Justification. In general, we felt that there are more similarities than differences between neonatal and pediatric DCD practice. As with all potential DCD donors, optimal EOL care should remain the fundamental concern in a neonatal pDCD process. The particular relational and ethical aspects of neonatal death require the expertise of a clinician trained to deal with these EOL issues $(80,81)$. Also, even less is known about the rate of the dying process after WLST in neonates and how that might affect completion of pDCD in this population, which should be discussed with surrogates during the consent process.

One of the potential differences between neonatal and other populations is the relatively large numbers of regional, nontertiary NICUs that do not offer pDCD (compared with relatively small number of PICUs) in which many potential neonatal pDCD donors may be initially hospitalized. If parents of children hospitalized in NICUs that do not offer pDCD wish to pursue $\mathrm{pDCD}$, clear protocols for transfer would be necessary, including consideration that the mother might not yet be eligible for transfer or discharge (82).

We recommend that pDCD can be offered to patients born with anencephaly or other similar severe, life-limiting neurologic disorders. In 2016, the Canadian Paediatric Society reaffirmed its position statement (83) that recommends against allowing deceased organ donation in this population based on the impossibility to complete a NDD examination in the setting of a functional brain stem. This statement, however, was based solely on NDD. Since pDCD is unaffected by the fact that these patients do not fulfill NDD criteria, we recommend that pDCD can be offered to the substitute decision makers of patients born with this condition.

\section{pDCD Implementation and Oversight Good Practice Statements.}

50) pDCD programs should seek out formal institutional approval within the existing hospital reporting structure.

51) There should be an integrated, collaborative approach to pDCD implementation with all hospital stakeholders, family and/or public partners, regional ODOs, and transplant programs.

52) Local coroners should be contacted early in the process of developing local $\mathrm{pDCD}$ procedures.

53) Communication and education of staff should be considered a priority during the development and implementation of a pDCD protocol.

54) pDCD case management review, including regular debriefing and a periodic quality assurance process, should occur.

55) Support for healthcare professionals involved in $\mathrm{pDCD}$ should be provided.

Justification. The establishment of a pDCD program should involve multidisciplinary collaboration with oversight from appropriate local authorities. The need for communication and education of all involved stakeholders has also been broadly emphasized in recent publications and was a frequently expressed sentiment during our pDCD symposium $(25,81)$. Quality control for this low-frequency, high-impact event is critical for $\mathrm{pDCD}$ programs. This process should involve medi$\mathrm{cal}$ and ethical oversight, ideally with linkage to measures of 
donor family experiences and transplant outcomes. Establishing robust ethical oversight also decreases the potential impact of institutional bias toward organ donation. Organ donation and transplantation activity are often a high-profile endeavor within a healthcare system, and the positive exposure associated with these programs may lead to a perception that donation activity takes precedence over ethical safeguards. The recognition and mitigation of this source of potential conflict interest is an important aspect of maintaining professional and public trust in the donation system.

Although we do not provide specific recommendation for documentation, inherent in the quality assurance component of recommendation 54 is the assumption that the process of pDCD be well documented. We encourage teams developing pDCD practices to visit the Canadian Blood Services website link listed below to see sample clinical and administrative checklists as well as documentation tools.

\section{CONCLUSION}

These recommendations are the result of a 3-year development process and represent the first pediatric-specific, national guideline governing $\mathrm{pDCD}$ practice. The process was transparent and based on the best available evidence that was synthesized into recommendations using the most rigorous methods possible. Review of this literature highlighted several knowledge gaps that hopefully will be addressed by further research in the field (5). For further information regarding our methods and the justifications behind our recommendations, please visit http://www.organsandtissues.ca/s/english-expert/ leading-practices-public-awareness-and-education for the full report in English and French, as well as tools to facilitate pDCD implementation. For questions regarding establishing a pDCD program, please contact Dr. Weiss or Canadian Blood Services.

Although no guideline can perfectly address all concerns held by all stakeholders, it is our sincere hope that application of these guidelines can increase the number of organs available to Canadians, while also offering the meaningful possibility of organ donation to families experiencing the loss of a child.

\section{ACKNOWLEDGMENTS}

Development of these guidelines would not have been possible without the tenacious administrative support from: Clay Gillrie, Lisa McCarthy-Tamblyn, Sylvia Torrance, Stephanie Currie-McCarragher, Debbie White, and Kerry Phillips. Jennifer Wolfsmith and Emilie Therien provided invaluable input as patient partners. Dr. Marcelo Cypel responded to questions regarding lung donation after circulatory determination of death (DCD). We sincerely appreciate and honor the gift made by deceased donors and their loved ones, including Frank Fontana and his family. Without their selflessness, organ donation and transplantation would not be possible. Members of the pediatric DCD (pDCD) Working Groups: The members of the pDCD guideline development were divided into the following working groups: 1) Ethics and Legal, 2) WLST, 3) Death Determination, 4) Eligibility, 5) Ante and post mortem interventions,
6) Neonatal, 7) Cardiac, and 8) the Planning Committee. Members and their groups were as follows: Amber Appleby (8), Jill Barter (2), Allon Beck $(2,4)$, Mary Bennet (cochair of 5), Daniel Buchman (cochair of 1), Kevin Coughlin (6), Alf Conradi (5), Cynthia Cupido (1), Roseanne Dawson (1), Sonny Dhanani (chair of 3), Anne Dipchand (7), Catherine Farrell (chair of 2), Darren Freed (7), Aviva Goldberg (chair of 4), Rebecca Greenberg (cochair of 1), Karen Hornby (4), Laura Hornby $(3,8)$, Valerie Langlois (4), Cheryl Mack (1), Meagan Mahoney (3), Deepak Manhas (6), Bram Rochwerg (8), Sam Shemie (cochair of 8), Ram Singh (cochair of 5), V. Ben Sivarajan (chair of 7), Christopher Tomlinson (3,6), Michael van Manen (2, chair of 6), Matthew Weiss (chair of 8), Samara Zavalkoff (5).

\section{REFERENCES}

1. Shemie SD, Baker AJ, Knoll G, et al: National recommendations for donation after cardiocirculatory death in Canada: Donation after cardiocirculatory death in Canada. CMAJ 2006; 175:S1

2. Canadian Institute for Health Information: Canadian Organ Replacement Register Annual Report: Treatment of End-Stage Organ Failure in Canada, 2004 to 2013. 2015. Available at: https://secure. cihi.ca/estore/productFamily.htm?locale=en\&pf=PFC2864\&lang=en. Accessed April 1, 2017

3. Martin DE, Nakagawa TA, Siebelink MJ, et al; Transplantation Society: Pediatric deceased donation-a report of the transplantation society meeting in Geneva. Transplantation 2015; 99:1403-1409

4. Antommaria $\mathrm{AH}$, Trotochaud K, Kinlaw K, et al: Policies on donation after cardiac death at children's hospitals: A mixed-methods analysis of variation. JAMA 2009; 301:1902-1908

5. Weiss MJ, Hornby L, Witteman W, et al: Pediatric donation after circulatory determination of death: A scoping review. Pediatr Crit Care Med 2016; 17:e87-e108

6. World Health Organization Guidelines Review Committee: WHO Handbook for Guideline Development, 2nd edition. 2014. Available at: http://www.who.int/kms/guidelines_review_committee/en/. Accessed January 1, 2015

7. Palda VA, Davis D, Goldman J: A guide to the Canadian Medical Association handbook on clinical practice guidelines. CMAJ 2007; 177:1221-1226

8. Davis D, Goldman J, Palda V: Canadian Medical Association Handbook on Clinical Practice Guidelines. Ottawa, ON, Canadian Medical Association, 2007

9. Schünemann HJ, Wiercioch W, Etxeandia I, et al: Guidelines 2.0: Systematic development of a comprehensive checklist for a successful guideline enterprise. CMAJ 2014; 186:E123-E142

10. Andrews JC, Schünemann HJ, Oxman AD, et al: GRADE guidelines: 15. Going from evidence to recommendation-determinants of a recommendation's direction and strength. J Clin Epidemiol 2013; 66:726-735

11. Guyatt $\mathrm{GH}$, Schünemann HJ, Djulbegovic B, et al: Guideline panels should not GRADE good practice statements. J Clin Epidemiol 2015; 68:597-600

12. Gries CJ, White DB, Truog RD, et al; American Thoracic Society Health Policy Committee: An official American Thoracic Society/ International Society for Heart and Lung Transplantation/Society of Critical Care Medicine/Association of Organ and Procurement Organizations/United Network of Organ Sharing Statement: Ethical and policy considerations in organ donation after circulatory determination of death. Am J Respir Crit Care Med 2013; 188: 103-109

13. Australia and New Zealand Intensive Care Society: The ANZICS Statement on Death and Organ Donation (Edition 3.2) Melbourne: ANZICS, 2013

14. The Intensive Care Society (UK) and the British Transplant Society: Donation after Circulatory Death - report on a consensus meeting. 2010. Available at: https://bts.org.uk/guidelines-standards/archivedguidelines/. Accessed July 1, 2014 
15. Downar J, Delaney JW, Hawryluck L, et al: Guidelines for the withdrawal of life-sustaining measures. Intensive Care Med 2016; 42:1003-1017

16. Committee on Bioethics: Ethical controversies in organ donation after circulatory death. Pediatrics 2013; 131:1021-1026

17. American Academy of Pediatrics Committee on Hospital Care: Policy statement--pediatric organ donation and transplantation. Pediatrics 2010; 125:822-828

18. Joffe AR, Carcillo J, Anton N, et al: Donation after cardiocirculatory death: A call for a moratorium pending full public disclosure and fully informed consent. Philos Ethics Humanit Med 2011; 6:17

19. Rady MY, Verheijde JL, McGregor J: Organ donation after cardiac death: Are we willing to abandon the dead-donor rule? Pediatr Crit Care Med 2007; 8:507; author reply 507-509

20. Setta SM, Shemie SD: An explanation and analysis of how world religions formulate their ethical decisions on withdrawing treatment and determining death. Philos Ethics Humanit Med 2015; 10:6

21. Nakagawa TA, Rigby MR, Bratton $S$, et al: A call for full public disclosure for donation after circulatory determination of death in children. Pediatr Crit Care Med 2011; 12:375-7; author reply 377

22. Ethics Committee American College of Critical Care Medicine Society of Critical Care Medicine: Recommendations for nonheartbeating organ donation. A position paper by the Ethics Committee, American College of Critical Care Medicine, Society of Critical Care Medicine. Crit Care Med 2001; 29:1826-1831

23. Bernat JL: The boundaries of organ donation after circulatory death. N Engl J Med 2008; 359:669-671

24. Costarino AT: Our continued unrealized potential for organ donation after cardiac death. Pediatr Crit Care Med 2009; 10:267-269

25. Kolovos NS, Webster P, Bratton SL: Donation after cardiac death in pediatric critical care. Pediatr Crit Care Med 2007; 8:47-49

26. Harrison $\mathrm{CH}$, Laussen PC: Controversy and consensus on pediatric donation after cardiac death: Ethical issues and institutional process. Transplant Proc 2008; 40:1044-1047

27. CSTCNTRP Increased Risk Donor Working Group: Guidance on the use of increased infectious risk donors for organ transplantation. Transplantation 2014; 98:365-369

28. Glazier AK: The principles of gift law and the regulation of organ donation. Transp/ Int 2011; 24:368-372

29. Brierley J: Neonatal organ donation: Has the time come? Arch Dis Child Fetal Neonatal Ed 2011; 96:F80-F83

30. Brierley J, Shaw D: Premortem interventions in dying children to optimise organ donation: An ethical analysis. J Med Ethics 2016; 42:424-428

31. Canadian Blood Services Deceased Donation: End-of-Life Conversations with Families of Potential Donors: Leading Practices in Offering the Opportunity for Donation. 2014. Available at: https://profedu.blood.ca/sites/msi/files/end-of-life_aug2014.pdf. Accessed January 1, 2015

32. Mulvania P, Mehakovic E, Wise C, et al: Successful international collaboration improves family donation conversations resulting in increased organ donation. Transplant Proc 2014; 46:2058-2065

33. Jansen NE, van Leiden HA, Haase-Kromwijk BJ, et al: Appointing 'trained donation practitioners' results in a higher family consent rate in the Netherlands: a multicenter study. Transp/ Int 2011; 24:1189-1197

34. Dubay DA, Redden DT, Haque A, et al: Do trained specialists solicit familial authorization at equal frequency, regardless of deceased donor characteristics? Prog Transplant 2013; 23:290-296

35. Siminoff LA, Gordon N, Hewlett J, et al: Factors influencing families' consent for donation of solid organs for transplantation. JAMA 2001; 286:71-77

36. Siminoff LA, Marshall HM, Dumenci L, et al: Communicating effectively about donation: An educational intervention to increase consent to donation. Prog Transplant 2009; 19:35-43

37. DeJong W, Franz HG, Wolfe SM, et al: Requesting organ donation: An interview study of donor and nondonor families. Am J Crit Care 1998; 7:13-23

38. Gortmaker SL, Beasley CL, Sheehy E, et al: Improving the request process to increase family consent for organ donation. J Transp/ Coord 1998; 8:210-217
39. Rodrigue JR, Cornell DL, Howard RJ: Attitudes toward financial incentives, donor authorization, and presumed consent among nextof-kin who consented vs. refused organ donation. Transplantation 2006; 81:1249-1256

40. Rosel J, Frutos MA, Blanca MJ, et al: Discriminant variables between organ donors and nondonors: A post hoc investigation. J Transp/ Coord 1999; 9:50-53

41. von Pohle WR: Obtaining organ donation: Who should ask? Heart Lung 1996; 25:304-309

42. Klieger J, Nelson K, Davis, R, et al: Analysis of factors influencing organ donation consent rates. J Transplant Coord 1994, 4:132-134

43. Salim A, Brown C, Inaba K, et al: Improving consent rates for organ donation: The effect of an inhouse coordinator program. J Trauma 2007; 62:1411-1414

44. ACRE Trial Collaborators: Effect of "collaborative requesting" on consent rate for organ donation: Randomised controlled trial (ACRE trial). BMJ 2009; 339:b3911

45. Ross LF, Frader J: Are we ready to expand donation after cardiac death to the newborn population? J Pediatr 2011; 158:6-8

46. Andrews PA, Burnapp L, Manas D; British Transplantation Society: Summary of the British Transplantation Society Guidelines for transplantation from Donors after deceased circulatory death. Transplantation 2014; 97:265-270

47. Murphy P, Boffa C, Manara A, et al: In-hospital logistics: What are the key aspects for succeeding in each of the steps of the process of controlled donation after circulatory death? Transp/ Int 2016; 29:760-770

48. Shore PM, Huang R, Roy L, et al: Development of a bedside tool to predict time to death after withdrawal of life-sustaining therapies in infants and children. Pediatr Crit Care Med 2012; 13:415-422

49. Shemie SD, Hornby L, Baker A, et al; The International Guidelines for Determination of Death phase 1 participants, in collaboration with the World Health Organization: International guideline development for the determination of death. Intensive Care Med 2014; 40:788-797

50. Youngner SJ, Arnold RM: Ethical, psychosocial, and public policy implications of procuring organs from non-heart-beating cadaver donors. JAMA 1993; 269:2769-2774

51. Verheijde JL, Rady MY: Pediatric organ donation and transplantation policy statement: More questions, not answers. Pediatrics 2010; 126:e489-e491; author reply e492

52. Veatch RM: Donating hearts after cardiac death - reversing the irreversible. N Engl J Med 2008; 359:672-673

53. Bernat JL, Capron AM, Bleck TP, et al: The circulatory-respiratory determination of death in organ donation. Crit Care Med 2010; 38:963-970

54. Tibballs J, Russell P: Reliability of pulse palpation by healthcare personnel to diagnose paediatric cardiac arrest. Resuscitation 2009; 80:61-64

55. Tibballs J, Weeranatna C: The influence of time on the accuracy of healthcare personnel to diagnose paediatric cardiac arrest by pulse palpation. Resuscitation 2010; 81:671-675

56. Eberle B, Dick WF, Schneider T, et al: Checking the carotid pulse check: Diagnostic accuracy of first responders in patients with and without a pulse. Resuscitation 1996; 33:107-116

57. Hornby K, Hornby L, Shemie SD: A systematic review of autoresuscitation after cardiac arrest. Crit Care Med 2010; 38:1246-1253

58. Dhanani S, Hornby L, Ward R, et al: Vital signs after cardiac arrest following withdrawal of life-sustaining therapy: A multicenter prospective observational study. Crit Care Med 2014; 42:2358-2369

59. de Vries EE, Hoogland PE, Wind J, et al: Transplantation of kidneys from paediatric DCD donors: A comparison with DBD donors. Nephrol Dial Transplant 2013; 28:220-226

60. Hong JC, Venick R, Yersiz H, et al: Liver transplantation in children using organ donation after circulatory death: A case-control outcomes analysis of a 20-year experience in a single center. JAMA Surg 2014: 149:77-82

61. Mason DP, Murthy SC, Gonzalez-Stawinski GV, et al: Early experience with lung transplantation using donors after cardiac death. $J$ Heart Lung Transplant 2008; 27:561-563

62. Kamal L, Lindower J, Kayler LK: Outcomes of kidney transplant recipients from donation after circulatory death donors without preagonal heparin administration. Transplantation 2015; 99:e167-e168 
63. Cypel M, Levvey B, Van Raemdonck D, et al: Favorable outcomes of donation after cardiac death in lung transplantation: A multicenter study. J Heart Lung Transplant 2013; 32:S15

64. Rojas-Peña A, Sall LE, Gravel MT, et al: Donation after circulatory determination of death: The university of michigan experience with extracorporeal support. Transplantation 2014; 98:328-334

65. Oniscu GC, Randle LV, Muiesan P, et al: In situ normothermic regional perfusion for controlled donation after circulatory death-the United Kingdom experience. Am J Transplant 2014; 14:2846-2854

66. Jiménez-Galanes S, Meneu-Diaz MJ, Elola-Olaso AM, et al: Liver transplantation using uncontrolled non-heart-beating donors under normothermic extracorporeal membrane oxygenation. Liver Transp/ $2009 ; 15: 1110-1118$

67. Fondevila C, Hessheimer AJ, Flores E, et al: Applicability and results of Maastricht type 2 donation after cardiac death liver transplantation. Am J Transplant 2012; 12:162-170

68. Otero A, Gómez-Gutiérrez M, Suárez F, et al: Liver transplantation from Maastricht category 2 non-heart-beating donors. Transplantation 2003; 76:1068-1073

69. Pelletier SJ, Hundley JC, Englesbe MJ, et al: Liver transplantation and ECMO-assisted donation after cardiac death [abstract]. Am J Transplant 2009; 9(Suppl 2):263

70. Sánchez-Fructuoso Al, Marques M, Prats D, et al: Victims of cardiac arrest occurring outside the hospital: A source of transplantable kidneys. Ann Intern Med 2006; 145:157-164

71. Valero R, Cabrer C, Oppenheimer F, et al: Normothermic recirculation reduces primary graft dysfunction of kidneys obtained from non-heartbeating donors. Transpl Int 2000; 13:303-310

72. Reznik O, Skvortsov A, Loginov I, et al: Kidney from uncontrolled donors after cardiac death with one hour warm ischemic time: Resuscitation by extracorporal normothermic abdominal perfusion "in situ" by leukocytes-free oxygenated blood. Clin Transplant 2011; 25:511-516
73. Magliocca JF, Magee JC, Rowe SA, et al: Extracorporeal support for organ donation after cardiac death effectively expands the donor pool. J Trauma 2005; 58:1095-1101; discussion 1101-1102

74. Farney AC, Singh RP, Hines $M H$, et al: Experience in renal and extrarenal transplantation with donation after cardiac death donors with selective use of extracorporeal support. J Am Coll Surg 2008; 206:1028-1037

75. Riou B, Guesde R, Jacquens $Y$, et al: Fiberoptic bronchoscopy in brain-dead organ donors. Am J Respir Crit Care Med 1994; 150:558-560

76. Cypel M, Sato M, Yildirim E, et al: Initial experience with lung donation after cardiocirculatory death in Canada. J Heart Lung Transplant 2009; 28:753-758

77. Alvarez A, Moreno P, Espinosa D, et al: Assessment of lungs for transplantation: A stepwise analysis of 476 donors. Eur J Cardiothorac Surg 2010; 37:432-439

78. Boucek MM, Mashburn C, Dunn SM, et al; Denver Children's Pediatric Heart Transplant Team: Pediatric heart transplantation after declaration of cardiocirculatory death. N Engl J Med 2008; 359:709-714

79. Dhital KK, lyer A, Connellan M, et al: Adult heart transplantation with distant procurement and ex-vivo preservation of donor hearts after circulatory death: A case series. Lancet 2015; 385:2585-2591

80. Brierley J, Larcher V: Organ donation from children: Time for legal, ethical and cultural change. Acta Paediatr 2011; 100:1175-1179

81. Devictor D: Organ donation after cardiac death: The subtle line between patient and donor care. Pediatr Crit Care Med 2007; 8:290-291

82. Labrecque M, Parad R, Gupta M, et al: Donation after cardiac death: The potential contribution of an infant organ donor population. J Pediatr 2011; 158:31-36

83. Canadian Paediatric Society Bioethics Committee: Use of anencephalic newborns as organ donors. Paediatr Child Health 2005; 10:335-337 